\title{
Combination of Clindamycin and Azithromycin as Alternative Treatment for Toxoplasma gondii Encephalitis
}

\section{Daisuke Shiojiri, Ei Kinai, Katsuji Teruya, Yoshimi Kikuchi, Shinichi Oka}

Author affiliation: National Centre for Global Health and Medicine, Tokyo, Japan

DOI: https://doi.org/10.3201/eid2504.181689

Current standard therapies for toxoplasmic encephalitis often cause severe adverse events. A 57-year-old HIV-positive man in Japan who had toxoplasmic encephalitis but was intolerant to trimethoprim/sulfamethoxazole, pyrimethamine, sulfadiazine, and atovaquone was successfully treated with the combination of clindamycin and azithromycin. This drug combination can be an alternative treatment for this condition.

$\mathrm{C}$ urrent treatment guidelines for toxoplasmic encephalitis (TE) (1) recommend either pyrimethamine plus sulfadiazine or pyrimethamine plus clindamycin; trimethoprim/sulfamethoxazole is also known to have comparable potency (2). However, kidney, liver, and hematologic toxicity, as well as hypersensitivities, often have been reported (2), and few alternatives are available. We report a TE patient who experienced severe toxicity after all standard regimens but was successfully treated with a combination of clindamycin and azithromycin.

A 57-year-old man sought care at the National Centre for Global Health and Medicine (Tokyo, Japan) with fever and mild disorientation. On examination, he was febrile and drowsy with slurred speech. He did not exhibit any apparent focal neurologic deficits.

Blood laboratory test results (reference ranges) were as follows: hemoglobin, $10.4 \mathrm{~g} / \mathrm{dL}(13.7-16.8 \mathrm{~g} / \mathrm{dL})$; total leukocyte count, $6.81 \times 10^{3}$ cells $/ \mathrm{L}\left(3.3-8.6 \times 10^{3}\right.$ cells $/ \mathrm{L})$; platelet count, $2.43 \times 10^{5} / \mathrm{L}\left(1.58-3.48 \times 10^{5} / \mathrm{L}\right)$; $\beta$-D-glucan, $118.4 \mathrm{pg} / \mathrm{mL}(<20.0 \mathrm{pg} / \mathrm{mL})$; CD4 ${ }^{+}$count, 58 cells $/ \mu \mathrm{L}(500-1,500$ cells $/ \mu \mathrm{L})$; and HIV RNA, $5.1 \times 10^{5}$ copies/mL (undetected). Brain magnetic resonance imaging results indicated 3 lesions with high intensity on T2weighted images and irregular ring enhancement. The lesions were in the right frontal lobe (diameter $15.6 \mathrm{~mm}$ ), left globus pallidus (diameter $17.5 \mathrm{~mm}$ ), and body of the caudate nucleus extending to the left thalamus (diameter $16.2 \mathrm{~mm}$; Figure, panel A). For cerebrospinal fluid (CSF), laboratory test results (reference ranges) were as follows: leukocyte count 0.3 cells $/ \mathrm{mm}^{3}\left(0-5\right.$ cells $\left./ \mathrm{mm}^{3}\right)$; protein, 39 $\mathrm{mg} / \mathrm{dL}$ (15.8-52.6 mg/dL); glucose, $54 \mathrm{mg} / \mathrm{dL}$ (47-69 mg/ $\mathrm{dL}$ ); and adenosine deaminase, $2.2 \mathrm{U} / \mathrm{L}(0-20 \mathrm{U} / \mathrm{L})$. Results of real-time PCR were negative for Mycobacterium, Epstein-Barr virus, cytomegalovirus, herpes simplex virus, and JC virus in CSF. Despite mild elevation of $\beta$-D-glucan, results of cryptococcal antigen testing were negative. No brain biopsy was performed. Although serum toxoplasma IgM and IgG titers were initially negative, IgG seroconversion was observed in serial tests 6 months $(9.2 \mathrm{IU} / \mathrm{mL})$ and 12 months $(39.7 \mathrm{IU} / \mathrm{mL})$ later. Toxoplasma nucleic acid in CSF was detected using a loop-mediated isothermal amplification technique (LAMP) with previously validated methods (3).

Trimethoprim/sulfamethoxazole $(10 \mathrm{mg} / \mathrm{kg} / \mathrm{d}$ trimethoprim) was first initiated because of limited availability of pyrimethamine plus sulfadiazine at the time, but acute liver toxicity developed on treatment day 6 (increases in levels of aminotransferase/alanine aminotransferase from 24/20 IU/L to 127/145 IU/L[(reference 10-42 IU/L]). Pyrimethamine plus sulfadiazine was started on treatment day 7. However, on day 9, acute renal failure developed because of obstructive urolithiasis caused by sulfadiazine crystals. After the patient was switched to pyrimethamine plus clindamycin, his renal function persistently worsened, and a drug-induced lymphocyte stimulation test suggested that pyrimethamine was responsible for the acute renal failure. A switch to atovaquone on treatment day 12 resulted in thrombocytopenia by day 17 .

After switching to the combination of clindamycin $(2,400 \mathrm{mg} / \mathrm{d})$ and azithromycin $(1,200 \mathrm{mg} / \mathrm{d})$, the patient did not experience any apparent adverse effects or show abnormal laboratory values. He successfully started antiretroviral therapy for his HIV infection during week 6 . Repeated brain magnetic resonance imaging performed 1, 3 , and 8 months after presentation showed all lesions had substantially decreased, and only a residual nonenhancing lesion was observed 12 months after presentation (Figure, panels B-D). The patient has continued maintenance therapy with the same dose of clindamycin and azithromycin without any symptoms of relapse or immune reconstitution inflammatory syndrome.

Several reports have documented the high prevalence of various adverse events associated with the aforementioned standard therapies for TE $(2,4)$, but little information is available regarding the efficacy of alternative drugs. Although atovaquone is the only safe alternative agent for which efficacy and safety have been relatively well established, it has been proven to be less potent than standard therapies (5).

Clindamycin has in vitro activity against Toxoplasma gondii, inhibitory effects in vivo at lower concentrations (6), and the ability to penetrate into CSF (7), suggesting that it is a promising alternative agent for TE treatment. More important, 

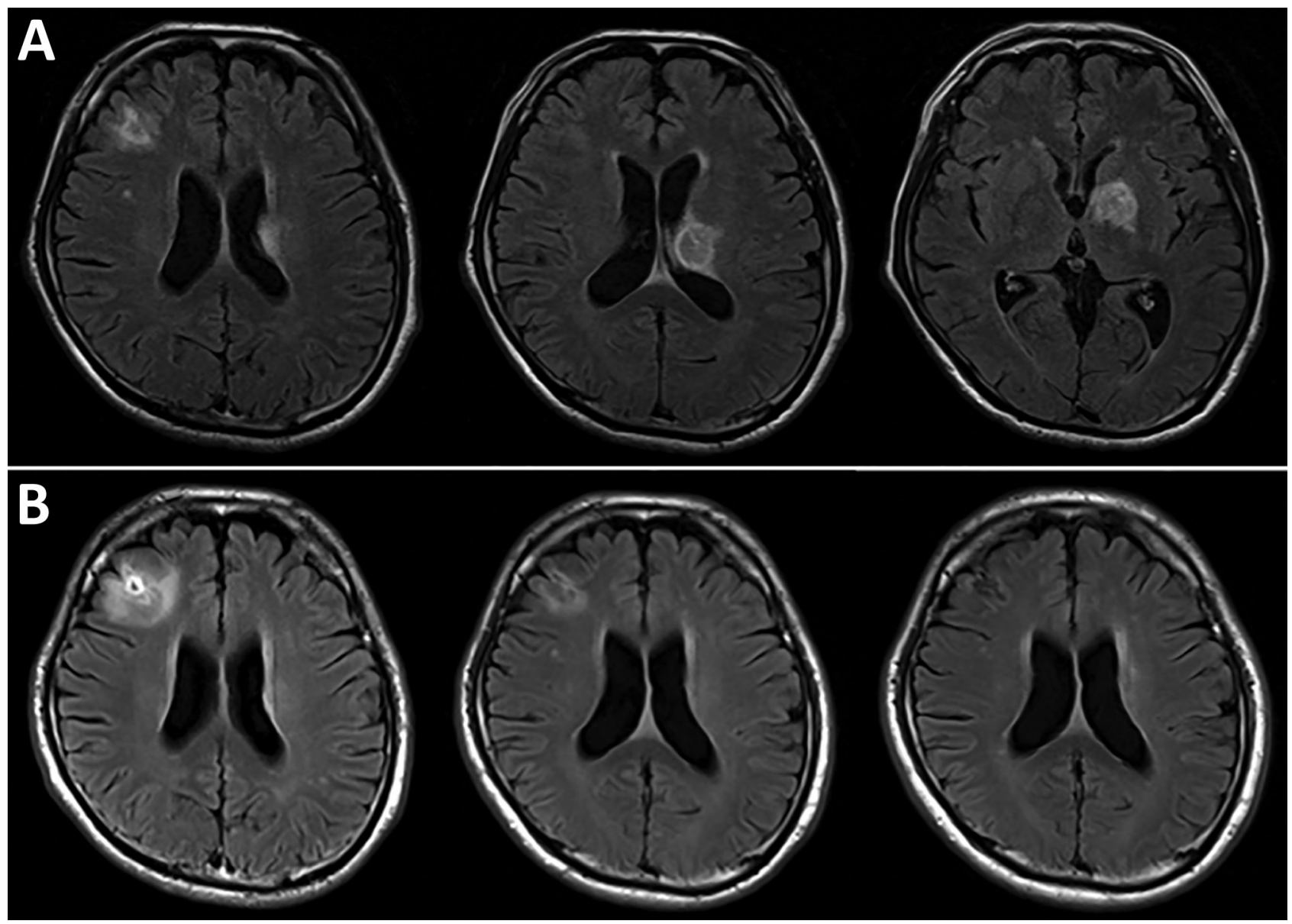

Figure. Serial brain magnetic resonance imaging results for a 57-year-old man with Toxoplasma gondii encephalitis, Tokyo, Japan.

A) All 3 lesions were evident when the patient first sought care. B) Chronologic changes are shown of the lesion in the right frontal lobe in response to antitoxoplasmic therapy after 1 (left), 3 (center), and 12 (right) months.

clindamycin causes far fewer adverse events than sulfadiazine $(2,4)$. Although the potency of clindamycin monotherapy has not been assessed, some case reports suggested its effectiveness (8). Therefore, a clindamycin-containing combination with potent agents other than pyrimethamine may be a reasonable treatment option for TE treatment.

Macrolides also have displayed substantial in vitro efficacy against $T$. gondii (9). A phase I/II dose-escalation study of oral azithromycin in combination with pyrimethamine revealed the relative efficacy and safety of the regimen (10). Azithromycin combined with a potent agent other than pyrimethamine, such as clindamycin, can be theoretically expected to be a safe and effective option. Further study should be conducted to establish safer treatment options for TE.

The continuous reduction in the size of the lesion in the patient we report was partly explained by his good immune recovery. Our results suggest that the combination of clindamycin and macrolides could be a safer and potent alternative therapy for patients who are intolerant of current standard regimens.

\section{Acknowledgments}

We thank Tokio Hoshina for detecting Toxoplasma nucleic acid by LAMP.

\section{About the Author}

Dr. Shiojiri is a fellow at the AIDS Clinical Centre, National Centre for Global Health and Medicine, Tokyo, Japan. His primary research interests include anal human papillomavirus infection and anal precancer among men who have sex with men in Japan.

\section{References}

1. US Department of Health and Human Serivces. Guidelines for the prevention and treatment of opportunistic infections in HIVinfected adults and adolescents [cited 2018 Aug 23]. https://aidsinfo.nih.gov/guidelines

2. Hernandez AV, Thota P, Pellegrino D, Pasupuleti V, Benites-Zapata VA, Deshpande A, et al. A systematic review and meta-analysis of the relative efficacy and safety of treatment regimens for HIV-associated cerebral toxoplasmosis: is trimethoprim-sulfamethoxazole a real option? HIV Med. 2017;18:115-24. http://dx.doi.org/10.1111/hiv.12402 
3. Fallahi S, Seyyed Tabaei SJ, Pournia Y, Zebardast N, Kazemi B. Comparison of loop-mediated isothermal amplification (LAMP) and nested-PCR assay targeting the RE and $\mathrm{B} 1$ gene for detection of Toxoplasma gondii in blood samples of children with leukaemia. Diagn Microbiol Infect Dis. 2014;79:347-54. http://dx.doi.org/10.1016/j.diagmicrobio.2014.02.014

4. Katlama C, De Wit S, O’Doherty E, Van Glabeke M, Clumeck N; The European Network for Treatment of AIDS (ENTA) Toxoplasmosis Study Group. Pyrimethamine-clindamycin vs. pyrimethamine-sulfadiazine as acute and long-term therapy for toxoplasmic encephalitis in patients with AIDS. Clin Infect Dis. 1996;22:268-75. http://dx.doi.org/10.1093/clinids/22.2.268

5. Katlama C, Mouthon B, Gourdon D, Lapierre D, Rousseau F; Atovaquone Expanded Access Group. Atovaquone as long-term suppressive therapy for toxoplasmic encephalitis in patients with AIDS and multiple drug intolerance. AIDS. 1996;10:1107-12.

6. Fichera ME, Bhopale MK, Roos DS. In vitro assays elucidate peculiar kinetics of clindamycin action against Toxoplasma gondii. Antimicrob Agents Chemother. 1995;39:1530-7. http://dx.doi.org/10.1128/AAC.39.7.1530

7. Gatti G, Malena M, Casazza R, Borin M, Bassetti M, Cruciani M. Penetration of clindamycin and its metabolite N-demethyl- clindamycin into cerebrospinal fluid following intravenous infusion of clindamycin phosphate in patients with AIDS. Antimicrob Agents Chemother. 1998;42:3014-7. http://dx.doi.org/10.1128/ AAC.42.11.3014

8. Yapar N, Erdenizmenli M, Oğuz VA, Cakir N, Yüce A. Cerebral toxoplasmosis treated with clindamycin alone in an HIV-positive patient allergic to sulfonamides. Int J Infect Dis. 2005;9:64-6. http://dx.doi.org/10.1016/j.ijid.2004.05.004

9. Neville AJ, Zach SJ, Wang X, Larson JJ, Judge AK, Davis LA, et al. Clinically available medicines demonstrating anti-toxoplasma activity. Antimicrob Agents Chemother. 2015;59:7161-9. http://dx.doi.org/10.1128/AAC.02009-15

10. Jacobson JM, Hafner R, Remington J, Farthing C, Holden-Wiltse J, Bosler EM, et al.; ACTG 156 Study Team. Dose-escalation, phase I/II study of azithromycin and pyrimethamine for the treatment of toxoplasmic encephalitis in AIDS. AIDS. 2001;15:583-9. http://dx.doi.org/10.1097/00002030-200103300-00007

Address for correspondence: Daisuke Shiojiri, AIDS Clinical Centre, National Centre for Global Health and Medicine, 1-21-1, Toyama,

Shinjuku-ku, Tokyo 192-8655, Japan; email: dshiojiri@hosp.ncgm.go.jp

\section{Prion Plasmodium knowlesi cholera tularemia} P1010nS Eptesicus fuscus syncytium Klebsiella Leptospira sapovirus yaws Rickettsia Vibrio Chagas disease lotavilus Lyssavirus botulism Escherichia coli syphilis knemidocoptic mange Babesia hemozoin Campylobacter Acinetobacter Nyphilis knemidlocoptic mange Babesia hemozoin
Naegleria fowlerii Ehrlichia Leishmaniasis
Anopheles
Verona integron
Herpesirinus vaccination
Borna disease virus Borna disease virus Eloola Franciscella tularensis typhus Rickettsia orf Coxiella burnetii kobuvirus Orientia tsutsugamushi Norovirus tuberculosis Malaria measles quarantine Vange
Chikungunya pertactin Borrelia Leprosy. Bocavirus Candida Aspergillus quarantine Mange Brucella Calcivirus quarantine Peste des petits ruminants influenza

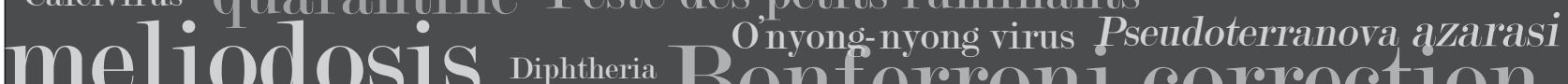
meliodosis pirnturin Bonferroni correction pertussis $\mathrm{V}$ elKe] celis Iginatzschineria Glanders Jersinia featured EMERCING monthly in INFECTIOUS DISEASES http://wwwnc.cdc.gov/eid/articles/etymologia 